\section{Facilitation of an extradimensional shift with overtraining in rats*}

\author{
T. GARY WALLER \\ University of Waterloo, Waterloo, Ont., Canada
}

In two experiments rats were either trained to criterion or were given overtraining beyond criterion on an orientation discrimination (left vs right oblique stripes). Ss in Experiment 1 were then transferred to a discrimination between two-dimensional shapes (cross vs triangle); $S$ s in Experiment 2 were transferred to a width discrimination (wide vs narrow vertical stripes). Position of the positive cue was the only variable irrelevant dimension in either phase of either experiment. Overtraining on the orientation discrimination improved performance on the shape discrimination but did not affect performance on the width discrimination. Several explanations were offered and implications for attention theories of discrimination learning were discussed.

The effect of overtraining on extradimensional (ED) and intradimensional (ID) shifts has been one of the experimental manipulations frequently used to test predictions from two-process theories of discrimination learning, such as Sutherland's stimulus-analyzer theory (Sutherland, 1964) or similar theories utilizing an attention construct (e.g., Zeaman \& House, 1963). Recent research (e.g., Shepp \& Turrisi, 1969) has shown, as predicted by the above theories, that overtraining facilitates performance on ID shifts but hinders performance on ED shifts. Typically, studies of ID and ED shifts begin with discrimination problems in which one dimension (Dimension A) is relevant, and at least one other dimension (Dimension B) is variable irrelevant (cf. Shepp \& Turrisi, 1966, 1969). During the shift phase, Dimension B is typically made relevant, and Dimension $A$, the dimension relevant during the original acquisition discrimination, is made variable irrelevant.

The prediction by stimulus-analyzer and similar theories that overtraining will hinder performance on ED shifts is not restricted to situations where Dimension $A$ is made variable irrelevant during transfer. The basis of the prediction that overtraining will hinder performance on an ED shift is that overtraining on Dimension A should strengthen the stimulus analyzer for Dimension A. Stimulus-analyzer theory stipulates that when one analyzer is strengthened, other analyzers are weakened. Although the predictions vary for different models (cf. Trabasso \& Bower, 1968), the indirect implication for stimulus-analyzer theory is

* This research was supported by Grant No. APA-326 from the National Research Council of Canada and by a grant from the University of Waterloo Research Council. that all other analyzers are weakened. Thus, overtraining not only strengthens relevant analyzers, it also weakens all other analyzers. During transfer, when Dimension B ceases to be irrelevant and becomes relevant, the probability of attending to Dimension $B$ should be diminished by previous overtraining on Dimension $\mathrm{A}$ whether or not Dimension A becomes variable irrelevant during shift. Thus, according to attention theories as they currently exist, overtraining should hinder performance on an ED shift whether or not Dimension A becomes variable irrelevant during shift. reported here was to examine the effect of overtraining on ED shifts when there were no variable-irrelevant cues (other than position) either during acquisition or shift. In two experiments rats were first trained on a stripe-orientation discrimination (left oblique vs right oblique) and were then trained on an ED shift with new arbitrarily selected stimuli. In Experiment 1, Ss were shifted to a discrimination between two-dimensional shapes (cross vs triangle); in Experiment 2 they were shifted to a stripe-width discrimination (wide vs narrow vertical stripes).

\section{EXPERIMENT 1}

Subjects

The Ss were 16 experimentally naive male albino rats supplied by the Holtzman Rat Company. Ss were reported to be approximately 100 days old on arrival at the laboratory.

\section{Apparatus}

The apparatus was a gray, wooden, Plexiglas-covered discrimination box. Start, choice, and goal areas were separated by horizontally sliding wooden doors. The start section was $51 / 2$ in. wide $\times 8$ in. long and led into the choice section, which was $8 \mathrm{in}$. long and triangle-shaped, increasing in width from 4 in. at the entrance to $12 \frac{1}{2}$ in. at the exit. Both start and choice sections
The purpose of the two experiments were $51 \%$ in. deep. The two adjacent goalboxes were each 6 in. wide. 91 in. long and $8 \mathrm{in}$. deep. The floor of the goalboxes was $2 \frac{1}{2}$ in. below the floor of the choice area. In the rear wall of each goalbox was a round hole, $1 \frac{1 / 2}{2}$. in diam, centered $1-5 / 8$ in. above the floor of the goalboxes. Food pellets were placed in 1 -in. foodwells located behind swinging doors that covered the holes. To get the reward pellets. $S$ had to put his head through the hole, pushing open the hinged door. The hinged doors in either goalbox could be locked from the outside.

Stimulus inserts for the rear walls of the goalboxes were cut from Masonite and

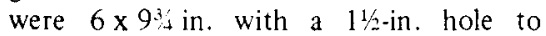
match the hole in the rear wall of the goalboxes. Two pairs of stimulus inserts were used. Each insert in the first pair was painted with alternating 1 -in. black and white stripes. On one insert the stripes were slanted at 45 deg from vertical to the left; on the other insert the stripes were slanted to the right. The stripes covered the entire stimulus insert. The second pair of inserts consisted of an equilateral triangle 5 in. on each side with the apex down, and a white symmetrical cross with $5 \times 1$ in. arms crossed at the center. Both geometrical figures were white on a black background. Each figure was laterally centered on the insert and was vertically positioned such that its lowest point was $1 \mathrm{in}$. above the top edge of the hole.

Procedure

On arrival $\mathrm{Ss}$ were housed individually and given ad lib food and water for $2-4$ days. Then they were placed on a once-daily ration of $10-12 \mathrm{~g}$ of laboratory cubes to maintain a constant body weight for the remainder of the experiment. Body weight was maintained at $80 \%$ of ad lib weight $\pm 15 \mathrm{~g}$ for each $\mathrm{S}$. Following the ad lib period each $S$ received 6.8 days of prehandling. On each day each $\mathrm{S}$ was permitted to explore the top of a large gray table for $2 \mathrm{~min}$, was picked up and replaced at least three times by $E$, and was permitted access to five $.045 \mathrm{~g}$ Noyes pellets located in a glass dish in the center of the table.

Following prehandling, each $S$ received acquisition training on a simultaneous discrimination with orientation of stripes (left slant or right slant) as the relevant cue. For half the Ss left was positive $(\mathrm{S}+)$ and right was negative (S-); for the other half the conditions were reversed. For each correct response $\mathrm{S}$ received five $.045 \mathrm{~g}$ Noyes pellets. $S$ was never permitted to correct an error. errors were never rewarded, and the swinging door to the fouu cup was locked in the S-goalbox. Ss were confined in the goalbox for $10 \mathrm{sec}$ on nonrewarded trials and until they ate the 
Table I

Mean Errors to Criterion During Acquisition and Transfer for Groups $\mathrm{C}$ and $\mathrm{OT}$ for Experiment 1 and Experiment 2

\begin{tabular}{cccc}
\hline & & \multicolumn{2}{c}{ Experimental Phase } \\
\cline { 3 - 4 } Experi- & & Acquisition & Transfer \\
\hline \multirow{2}{*}{1} & C & 50.00 & 31.50 \\
& OT & 40.12 & 5.13 \\
2 & C & 56.13 & 77.75 \\
& OT & 67.50 & 60.62 \\
\hline
\end{tabular}

pellets on rewarded trials. The position of $\mathrm{S}+$, right or left, was varied according to randomly selected Gellerman series. A modified correction procedure was used such that, if $S$ made an error, the position of St was not changed on the next trial. Ss were run each day until they make five correct responses. The within-day intertrial interval was approximately $10 \mathrm{sec}$. Acquisition training continued for each $S$ until he make 13 correct responses out of 15 consecutive trials.

When $S$ s reached acquisition criterion, they were assigned randomly to one of two treatment groups with the restriction that eight $\mathrm{Ss}$ were assigned to each group. Ss assigned to Group C (criterion) began training on the transfer discrimination on the day after they reached acquisition criterion. Ss assigned to Group OT (overtraining) received another 10 days (15 trials per day) of training on the original discrimination and then began training on the transfer discrimination.

On the transfer task (an ED shift with no variable irrelevant cues other than position), the relevant cues were the triangle and the cross. All procedures were appropriately counterbalanced such that within each group, for half the Ss trained with left as $S+$ in acquisition, the triangle was S+ during transfer, etc. All procedures during transfer were the same as during original acquisition.

\section{Results}

Mean errors to criterion for Groups C and OT for acquisition and transfer during Experiment 1 are shown in Table 1. Analysis by $t$ tests indicated that the two groups did not differ during acquisition $(t<1.0)$ but that overtraining on the stripe-orientation discrimination caused a significant improvement in performance on the ED shift to the shape discrimination $(\mathrm{t}=3.17, \mathrm{df}=14, \mathrm{p}<.01)$. Mean errors during 10 days of overtraining for Group OT was 19.62 , approximately two errors per day.

\section{EXPERIMENT 2}

Experiment 1 demonstrated improved performance on an ED shift as a result of overtraining on a prior discrimination. The purpose of Experiment 2 was to repeat the basic design of Experiment 1 but with a different ED problem. Following training to criterion or overtraining, Ss were shifted from the stripe-orientation discrimination to a discrimination based on the width of vertical alternating black and white stripes. Method

The Ss were 16 experimentally naive rats of the same description as in Experiment 1. The apparatus and all procedures were identical to Experiment 1, except that a different $E$ ran the Ss and different stimulus inserts were used for the transfer task. The relevant cues during transfer were the widths of vertical alternating black and white stripes. On one stimulus insert the stripes were wide $\left(1 \frac{1 / 2}{\mathrm{in}}\right.$.); on the other insert the stripes were narrow( $3 / 4$ in.). Again all appropriate counterbalancing was done such that, for example, for Ss trained with left orientation as $\mathrm{S}+$ during acquisition, half were trained with wide as $\mathrm{S}+$ and half were trained with narrow as $\mathrm{S}+$ during transfer.

Mean errors to criterion for Experiment 2 are shown in Table 1. Analysis by $\mathrm{t}$ tests indicated that Groups $\mathrm{C}$ and OT did not differ during original acquisition $(t<1.0)$, and that overtraining on the original discrimination did not have an effect on performance on the ED shift $(t<1.0)$. Mean errors during 10 days of overtraining for Group OT was 16.25, approximately two errors per day. A comparison of acquisition data from the two separate experiments indicated that, although acquisition performance was slightly better in Experiment 1 than in Experiment 2, the difference was not significant $\quad(t=1.42, \quad d f=28$ $.10<\mathrm{p}<.20$ ).

\section{DISCUSSION}

In two separate experiments it was found that overtraining significantly facilitated performance on an ED shift (Experiment 1) or had no effect on performance on an ED shift (Experiment 2). The facilitation found in Experiment 1 is clearly at variance with previous research which found the opposite effect (Shepp \& Turrisi, 1969), or found no effect at all (Tighe, 1965), but is in substantial agreement with other research (Eimas, 1966) which found a facilitation as reported here. The reason (or reasons) for such discrepancies in the results of previous research is not clear and there are many differences among the previous experiments; e.g., stimuli used, species of $\mathrm{Ss}$, presence or absence of variable irrelevant cues, and amount of overtraining given.

Both experiments reported here used the same type, age, and sex of Ss from the same suppliers, the same discrimination apparatus, the same motivational conditions, the same reward conditions, and the same procedures in all other respects that can be discerned by $E$. Two major differences exist between the two experiments. First, two different Es collected the data. Second, different cues were used during the shift or transfer phase of the two experiments: A white cross and triangle were used in Experiment 1 , and vertical stripes differing in width were used in Experiment 2.

There are several possible explanations for the discrepancy in the results of the two experiments. (1) The discrepancy is attributable to some unknown but important factor related to the different Es. However, acquisition performance was not affected by $E$. If the transfer results are attributable to the different Es, a more consistent pattern of results should occur if proper comparisons are made within the same future experiment. (2) The significant effect of overtraining in Experiment 1 is a Type I error and the effect cannot be consistently replicated. (3) The insignificant effect of overtraining in Experiment 2 is due to a Type II error, and with a more precise test (e.g., with a larger N) a significant effect would be found. (4) The discrepant outcomes of the two experiments are due to differences in difficulty of the two transfer discriminations. If so, when animals are shifted from Dimension A to Dimension B, the effect of overtraining should depend upon whether or not the discrimination on Dimension B is an easy or a difficult discrimination. For example, if animals are shifted from a stripe-width discrimination to a line-orientation discrimination, the effect of overtraining should depend on the difficulty of the orientation discrimination; i.e., on whether the two orientations are easy to discriminate (e.g., 90-deg separation) or difficult to discriminate (e.g., 10-deg separation). (5) A final possibility is that the obtained results are reliable; i.e., when there are no variable irrelevant cues, overtraining sometimes facilitates performance on an ED shift and sometimes does not. Further, a crucial factor in determining the effect of overtraining is the relationship between the sets of cues used in acquisition and in transfer. If the relationship between the training and transfer cues is an important determinant of the effects of overtraining, then a theoretical explanation of the relationship is required.

Stimulus-analyzer and attention theories (Sutherland, 1964; Zeaman \& House, 1963) have clearly specified that analyzers analyze dimensions or that animals attend to or observe dimensions. Perhaps, however, stimulus analysis in animals occurs in a hierarchical fashion (cf. Sutherland \& Andelman, 1967) or that 
various levels of analysis of visual information are possible. For example, orientation or stripes is clearly a dimension to which an animal might attend, or a source of information that an animal might use to solve a discrimination problem. Further, overtraining on an orientation discrimination might increase the probability that the animal, when confronted with new stimuli, will attend first to orientation information if it is available. Clearly, one (there are almost certainly others) of the distinguishing or distinctive features (cf. Gibson, 1969; Neisser, 1967; Sutherland, 1969) that makes triangles different from crosses is the orientation of the contours in the two figures. Thus, it is not surprising that overtraining on a stripe-orientation discrimination improves performance on a discrimination between patterns that can be distinguished on the basis of orientation cues. Further, since orientation information is useless in discriminating stripes of various widths but of the same orientation, it should not be surprising that overtraining on stripe orientation did not facilitate transfer to the discrimination based on line width.

The above hypothesis is admittedly speculative and is only one of several possible interpretations of the data. However, the hypothesis does generate predictions that render it clearly amenable to refutation. There should be some ED shifts that are facilitated by overtraining on stripe orientation and some that are not. For example, according to the above hypothesis, overtraining on stripe orientation should not facilitate ED-shift performance on discriminations based on information unrelated to slant; e.g., width of stripes, size of squares in checkerboard patterns, sizes of circles, etc. According to the above hypothesis, overtraining should hinder performance on ED shifts where orientation information is present and available to the organism but is irrelevant to the solution of the shift discrimination. This last procedure is the one most typically used in ED-shift experiments and is the type of design that has most frequently shown that overtraining hinders ED shifts (cf. Shepp \& Turrisi, 1966).

A final point concerns the effect of overtraining on the strengths of stimulus analyzers. The original hypothesis. within the framework of stimulus-analyzer theory, was that overtraining on orientation would strengthen an orientation analyzer and, consequently, weaken all other analyzers. Specifically, it was hypothesized that overtraining would hinder subsequent transfer performance on another dimension (shape or width) regardless of the fact that no irrelevant orientation cues were present during transfer. The results of the two experiments clearly did not support the original hypothesis. An alternative hypothesis, which requires a modification of stimulus-analyzer theory, is that overtraining on one dimension does not necessarily weaken stimulus analyzers for all other dimensions.

\section{RHFH.RH NCHS}

IIVAS. P. D. lffects of overtraining and age on intradimensional and extradimencional thit in children. Joumal of l yperimental child Pychology. 1966. 3. 348.355.

GIBSON. I. J. Principles of pereeptual leaming and derelopment Din York: Appleton-Century-Croft 1969

NEISSER, C. Cognitive psichologr. liw York: Appleton-Century-Crofts, 1967.

SHEPP. B. I... \& TLRRISI. I. D. Learning and transfer of mediating responses in discriminative learning. In $\mathrm{X}$. R. lllis (Fd.). International review of research in mental retardation. New York: Academic Press. 1966. Pp 85-121.

SHEPP. BRYAN E.. \& TLRRISI. IRANK D. Effects of overtraining on the acquisition of intradimensional and extradimensional shitt: Journal of Experimental Psychology. 1969. 82, $46-51$

SLTHERLAND, N. S. The learning of discrimination by animals. Fndeavour, 1964, 23. 148-152.

SLTHERLAND, N. S. Outlines of a theory of visual pattern recognition in animals and men. In R. II. Gilbert and N. S. Sutherland (Eds.), Animal discrimination learning. New York: Academic Press, 1969. Pp. 385-411.

SLTHERLAND, N. S., \& ANDELMAN, L. Learning with one and two cues. Psychonomic Science, 1967, 7, 107-108.

TIGHE, T. J. Effect of overtraining on reversal and extradimensional shifts. Journal of Experimental Psychology, 1965. 70, 13-17.

TRABASSO, T., \& BOWER, G. H. Attention in learning. New York: Wiley. 1968.

ZEAMAN, D., \& HOLSE, B. J. The role of attention in retardate discrimination learning. In N. R. Ellis (Ed.), Handbook in mental deficiency: Psychological theory and research. New York: McGraw-Hill. 1963. Pp. 159-223. 